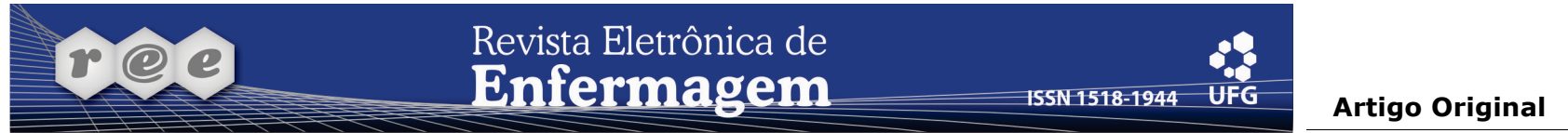

\title{
Perfil das internações de crianças em um centro de tratamento para queimados*
}

\author{
Profile of children hospitalized in a burn treatment center
}

Perfil de internaciones de niños en un centro de tratamiento del quemado

Priscila Santa de Moraes $^{1}$, Rosângela Aparecida Pimenta Ferrari ${ }^{2}$, Flávia Lopes Sant'Anna ${ }^{3}$, Jéssica Talita Mariana Wicthoff Raniero ${ }^{4}$, Laís da Silva Lima ${ }^{5}$,

Tabatha de Freitas Moreira Santos ${ }^{6}$, Mauren Teresa Grubisich Mendes Tacla ${ }^{7}$

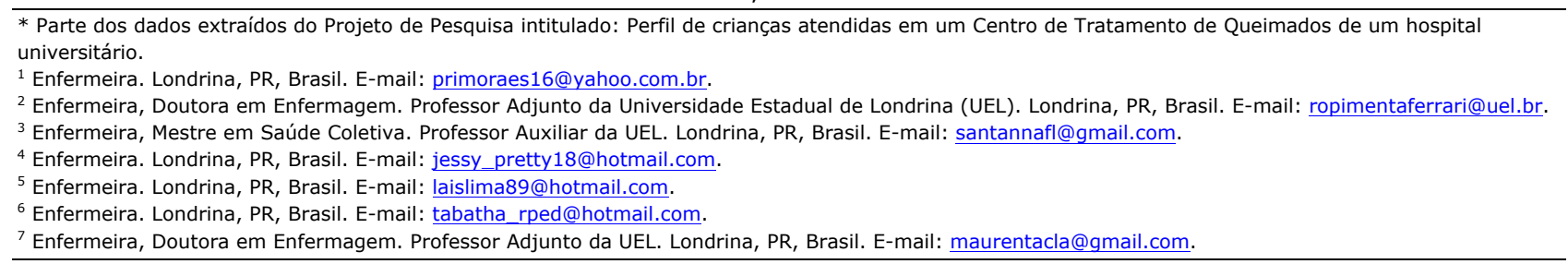

\section{RESUMO}

Estudo quantitativo, retrospectivo e transversal para caracterizar as internações pediátricas de um Centro de Tratamento para Queimados entre 2007 e 2011. Ocorreram 248 internações de crianças vítimas de queimadura, das quais $65,7 \%$ eram do sexo masculino e $50,4 \%$ com idade inferior a três anos. Aproximadamente $58,0 \%$ dos acidentes ocorreram no outono/inverno. O principal agente causador nos menores de três anos foi escaldadura (72,1\%) e, entre quatro e 12 anos, chama direta (52,5\%). 86,0\% das queimaduras foram classificadas como de $2^{\circ}$ e 30 graus, com predomínio nos membros superiores, tronco e cabeça. O tempo médio de internação foi de 14,8 dias. A alta melhorada ocorreu em $58,0 \%$ dos casos, 38,0\% necessitaram de encaminhamento para reabilitação e 4,0\% evoluíram a óbito. A queimadura na população infantil pode ser evitada por meio de investimentos em campanhas preventivas, principalmente para conscientização familiar sobre o risco de morbimortalidade infantil e sequelas por essa causa.

Descritores: Unidades de Queimados; Queimadura; Cuidado da Criança; Epidemiologia; Enfermagem Pediátrica.

\section{ABSTRACT}

A quantitative, retrospective and cross-sectional study to define the characteristics of pediatric hospitalization in a Burn Treatment Center between 2007 and 2011. There were 248 children burn victims hospitalized, of which $65.7 \%$ were male and $50.4 \%$ younger than three years of age. Approximately $58.0 \%$ of accidents occurred in autumn/winter. The main causative agent for those younger than three years was scalding $(72.1 \%)$, and for those between 4 and 12 years old, direct flame contact (52.5\%). $86.0 \%$ of burns were classified as second and third degree, predominately on upper limbs, torso and head. Mean hospitalization time was 14.8 days. Patients were discharged with improvement in $58.0 \%$ of cases, $38.0 \%$ were referred to rehabilitation and $4.0 \%$ died. Burns among the pediatric population can be avoided by investing in prevention campaigns, aimed especially at bringing awareness to families about the risk of child morbimortality and repercussions due to burns.

Descriptors: Burn Units; Burns; Child Care; Epidemiology; Pediatric Nursing.

\section{RESUMEN}

Estudio cuantitativo, retrospectivo, transversal, objetivando caracterizar las internaciones pediátricas de un Centro de Tratamiento del Quemado entre 2007 y 2011. Hubieron 248 internaciones de niños víctima de quemaduras, 65,7\% eran de sexo masculino, 50,4\% con edad inferior a tres años. Aproximadamente $58,0 \%$ de los accidentes sucedieron en otoño/invierno. El principal agente causante en menores de tres años fue la escaldadura $(72,1 \%)$, y entre 4 y 12 años la llama directa $(52,5 \%)$. El $86,2 \%$ de quemaduras fue clasificado como de $2^{\circ}$ y $3^{\circ}$ grado, con predominio en miembros superiores, tronco y cabeza. El tiempo promedio de internación fue de 14,8 días. El alta con mejoría se dio en $58,0 \%$ de casos, $38.0 \%$ necesitaron seguimiento para rehabilitación y $4,0 \%$ acabaron en fallecimientos. La quemadura en la población infantil puede evitarse mediante inversión en campañas preventivas, particularmente de concientización familiar sobre el riesgo de morbilidad y mortalidad infantil, y secuelas por dicha causa.

Descriptores: Unidades de Quemados; Quemaduras; Cuidado del Niño; Epidemiología; Enfermería Pediátrica. 


\section{INTRODUÇÃO}

Define-se queimadura a lesão habitualmente restrita à pele, decorrente da aplicação de calor ao corpo. Pode ser classificada de acordo com o tipo de agente causador (térmica, química ou elétrica) ou profundidade da lesão (primeiro, segundo ou terceiro graus) ${ }^{(1)}$.

A queimadura de primeiro grau acomete a epiderme e caracteriza-se por uma lesão úmida, hiperemiada, edemaciada e dolorosa ${ }^{(2)}$. Na de segundo grau há comprometimento tanto da epiderme quanto da derme, com formação de bolhas. A cicatrização é mais lenta e há chances de ocorrer sequelas como discromia ou cicatriz. Já na de terceiro grau há destruição da epiderme e da derme, podendo atingir tecido subcutâneo, tendões, ligamentos, músculos e ossos. Apresenta aspecto de couro esbranquiçado ou marmóreo ${ }^{(2-3)}$.

Nos Estados Unidos da América (EUA) a incidência anual dessa causa externa na faixa etária pediátrica, com necessidade de tratamento em serviços de urgência é de aproximadamente 120.000 casos, ou seja, 15 para cada 10.000 residentes $^{(4)}$. No Brasil, entre 1998 e 2007, ocorreram 297.729 internações por queimaduras no Sistema Único de Saúde (SUS), das quais 135.789 eram de menores de 14 anos. Dessas, 59,0\% compreenderam a faixa etária de zero a quatro anos e 25,0\% de cinco a nove $\operatorname{anos}^{(5)}$. As queimaduras são responsáveis pela morte direta ou indireta de mais de 300.000 pessoas no mundo e 2.500 no Brasil, a cada ano. Entre 1996 e 2011, dos 2.044.102 óbitos por essa causa, 339.986 foram entre menores de 20 anos e 48.240 tinham idade inferior a quatro anos ${ }^{(6)}$.

No período de desenvolvimento, a criança é curiosa, inquieta, inexperiente, exploradora, ativa e incapaz de identificar e avaliar o perigo, levando à maior prevalência de acidentes domésticos ${ }^{(7)}$. Na maioria dos casos o infante necessita de internação hospitalar em centros especializados, geralmente por longo tempo, aumentando ainda mais o custo hospitalar para o tratamento que exige inúmeras intervenções invasivas e acompanhamento para reabilitação da lesão após a alta $^{(8)}$.

O tratamento das queimaduras é um grande desafio não só pela gravidade das lesões, mas também pelas inúmeras complicações advindas dos tipos de agente causador e suscetibilidade às infecções ${ }^{(3)}$. Quando não levam à morte, podem ocasionar significativas limitações funcionais, psicológicas e sociais ${ }^{(9)}$. Complicações durante a internação de crianças implicam em sequelas por toda a vida, principalmente entre àquelas com idade precoce $^{(10-12)}$.

Nos EUA, aproximadamente quatro bilhões de dólares são gastos em tratamento e reabilitações. Já no Brasil há baixo investimento financeiro em centros especializados, limitando a oferta de serviços em relação ao número anual de vítimas ${ }^{(11)}$. Pesquisas epidemiológicas na área de queimaduras são importantes não apenas pela simples exposição de fatos e números, mas pela possibilidade de embasamento teórico para formulação de políticas e estratégias preventivas, haja vista a carência de trabalhos e de unidades especializadas. Assim, a presente pesquisa objetivou caracterizar as internações pediátricas em um Centro de Tratamento de Queimados (CTQ).

\section{MÉTODO}

Trata-se de um estudo quantitativo, retrospectivo e transversal, desenvolvido no CTQ do Hospital Universitário de Londrina (HUL), nível terciário, localizado na região Norte do Estado do Paraná/Brasil. Esse hospital conta com 316 leitos, e atende cerca de 250 municípios paranaenses e mais de 100 cidades de outros estados.

O CTQ do HUL, inaugurado em agosto de 2007, é o segundo do Estado. Oferece 16 leitos de internação, distribuídos em 10 de enfermaria e seis de Unidade de Terapia Intensiva (UTI), tanto para a população infantil quanto para a adulta.

A casuística do presente estudo compreendeu todas as crianças na faixa etária de zero a 12 anos internadas nessa unidade, entre agosto de 2007 a dezembro de 2011, totalizando 271. Foram excluídos 23 casos por não caracterizarem o objeto de estudo, restando 248 crianças. Utilizou-se como fonte de informação os prontuários disponibilizados pelo Sistema de Arquivos Médicos e Estatísticos (SAME) do hospital.

A coleta de dados foi realizada por meio de um formulário previamente testado contendo as seguintes variáveis: dados de identificação; período do dia da ocorrência do acidente; época do ano; agente causador; profundidade da queimadura; área queimada; contexto: eletricidade (fio desencapado, curto circuito e choque elétrico), superfície aquecida (ferro de passar, panela, forno e braseiro), escaldadura (café, leite, água e alimentos), chama direta (gasolina, álcool, tiner, incêndio e fogueira), churrasqueira (tentativa de acender ou estar próximo) e produtos químicos (soda cáustica); tempo de internação; e motivo da alta (melhorada, para reabilitação e óbito). 
Os dados foram processados em dupla entrada no programa Epi Info ${ }^{\circledR}$. A análise foi realizada utilizando estatística descritiva. Esta pesquisa foi submetida à análise e aprovação pelo Comitê de Ética em Pesquisa Envolvendo Seres Humanos da Universidade Estadual de Londrina com o Parecer de Aprovação no 032/2012 e CAAE no. 01048912.2.0000.5231.

\section{RESULTADOS}

Do total das 248 crianças internadas para o tratamento de queimadura no CTQ, 65,7\% eram do sexo masculino, 45,6\% tinham de um a três anos e $19,4 \%$ de quatro a seis anos. Quanto à procedência, 47,2\% eram de outras Regionais de Saúde, 37,1\% residiam no município de estudo e $14,9 \%$ pertenciam à Regional de Saúde de Londrina (Tabela 1).

Mais da metade das ocorrências tiveram como primeira causa a escaldadura por líquido superaquecido $(51,2 \%)$, seguida por chama direta $(30,8 \%)$. O período em que os acidentes foram mais frequentes foi 0 vespertino $(42,8 \%)$, e $57,8 \%$ dos casos aconteceram na estação outono/inverno (Tabela 2).

Entre as crianças menores de três anos a maioria das ocorrências foi por escaldadura $(72,1 \%)$. Na faixa etária de quatro a 12 predominou a chama direta $(52,5 \%)$ (Tabela 3).

Tabela 1: Caracterização sociodemográfica das crianças internadas no

Centro de Tratamento de Queimados, Londrina, PR, Brasil, 2007 a 2011.

\begin{tabular}{|c|c|c|}
\hline \multirow{2}{*}{ Caracterização Sociodemográfica } & \multirow{2}{*}{\begin{tabular}{|l}
$N$ \\
248 \\
\end{tabular}} & \multirow{2}{*}{\begin{tabular}{|l|}
$\%$ \\
100,0 \\
\end{tabular}} \\
\hline & & \\
\hline \multicolumn{3}{|l|}{ Sexo } \\
\hline Masculino & 163 & 65,7 \\
\hline Feminino & 85 & 34,3 \\
\hline \multicolumn{3}{|l|}{ Faixa Etária (em anos) } \\
\hline$<1$ & 12 & 4,8 \\
\hline 1 a 3 & 113 & 45,6 \\
\hline 4 a 6 & 48 & 19,4 \\
\hline 7 a 9 & 41 & 16,5 \\
\hline$<12$ & 34 & 13,7 \\
\hline \multicolumn{3}{|l|}{ Procedência } \\
\hline Londrina & 92 & 37,1 \\
\hline Regional de Saúde Londrina (17a) & 37 & 14,9 \\
\hline Outras Regionais de Saúde & 117 & 47,2 \\
\hline Outro Estado & 2 & 0,8 \\
\hline
\end{tabular}

Tabela 2: Tipo de ocorrência da queimadura segundo o período e época do ano, Londrina, PR, Brasil, 2007 a 2011.

\begin{tabular}{|c|c|c|c|c|c|c|c|c|c|c|}
\hline \multirow{4}{*}{ Tipo de ocorrência* } & \multicolumn{6}{|c|}{ Período } & \multicolumn{4}{|c|}{ Época do Ano } \\
\hline & \multicolumn{2}{|c|}{ Matutino } & \multicolumn{2}{|c|}{ Vespertino } & \multicolumn{2}{|c|}{ Noturno } & \multicolumn{2}{|c|}{ Primavera/Verão } & \multicolumn{2}{|c|}{ Outono/Inverno } \\
\hline & $\mathbf{n}$ & $\%$ & $\mathbf{n}$ & $\%$ & $\mathbf{n}$ & $\%$ & $n$ & $\%$ & $\mathbf{n}$ & $\%$ \\
\hline & 187 & 100,0 & 187 & 100,0 & 187 & 100,0 & 244 & 100,0 & 244 & 100,0 \\
\hline Eletricidade & 1 & 2,2 & 2 & 2,5 & 1 & 2,6 & 4 & 3,9 & 1 & 0,7 \\
\hline Superfície Aquecida & - & - & 5 & 6,3 & 2 & 3,2 & 7 & 6,8 & 8 & 5,7 \\
\hline Escaldadura & 28 & 62,2 & 36 & 45,0 & 37 & 59,7 & 49 & 47,6 & 76 & 53,9 \\
\hline Chama Direta & 11 & 24,4 & 28 & 35,0 & 20 & 32,3 & 34 & 33,0 & 46 & 32,6 \\
\hline Churrasqueira & 4 & 8,9 & 8 & 10,0 & 2 & 3,2 & 6 & 5,8 & 9 & 6,4 \\
\hline Químico & 1 & 2,2 & 1 & 1,3 & - & - & 3 & 2,9 & 1 & 0,7 \\
\hline TOTAL & 45 & 24,1 & 80 & 42,8 & 62 & 33,1 & 103 & 42,2 & 141 & 57,8 \\
\hline
\end{tabular}

Tabela 3: Tipo de agente causador da queimadura de acordo com a faixa etária, Londrina, PR, Brasil, 2007 a 2011.

\begin{tabular}{|c|c|c|c|c|}
\hline \multirow{4}{*}{ Tipo de agente causador* } & \multicolumn{4}{|c|}{ Faixa Etária (em anos) } \\
\hline & \multicolumn{2}{|c|}{1 a 3} & \multicolumn{2}{|c|}{4 a 12} \\
\hline & $\mathbf{n}$ & $\%$ & $\mathbf{n}$ & $\%$ \\
\hline & 244 & 100,0 & 244 & 100,0 \\
\hline Eletricidade & 1 & 0,8 & 4 & 3,3 \\
\hline Superfície Aquecida & 10 & 8,2 & 5 & 4,1 \\
\hline Escaldadura & 88 & 72,1 & 37 & 30,3 \\
\hline Chama Direta & 16 & 13,1 & 64 & 52,5 \\
\hline Churrasqueira & 4 & 3,3 & 11 & 9,0 \\
\hline Químico & 3 & 2,5 & 1 & 0,8 \\
\hline TOTAL & 122 & 50,0 & 122 & 50,0 \\
\hline
\end{tabular}

* Excluídos registros com informações ignoradas 
Os agentes térmicos foram os principais causadores de queimaduras $(96,0 \%)$, e aproximadamente $86,0 \%$ delas foram classificadas como sendo de $2^{\circ}$ e $2 \% / 3^{\circ}$ graus (Tabela 4).

Os membros superiores foram as regiões do corpo mais afetadas $(65,3 \%)$, seguido por tronco $(58,9 \%)$ e cabeça $(46,8 \%)$. As vias aéreas foram menos acometidas $(3,2 \%)$ (Tabela 5 ). A média de regiões corpóreas atingidas foi 2,5 por criança.

Tabela 4: Agente causador e profundidade/grau das queimaduras, Londrina, PR, Brasil, 2007 a 2011.

\begin{tabular}{|c|c|c|c|c|c|c|}
\hline \multirow{4}{*}{ Agente Causador } & \multicolumn{6}{|c|}{ Profundidade/Grau } \\
\hline & \multicolumn{2}{|c|}{$1^{\circ}$ e $10 / 2^{\circ}$} & \multicolumn{2}{|c|}{$2^{\circ}$ e $2^{\circ} / 3^{\circ}$} & \multicolumn{2}{|c|}{$3^{\circ}$ e $1^{\circ} / 2^{\circ} 3^{\circ}$} \\
\hline & $\mathbf{n}$ & $\%$ & $\mathbf{n}$ & $\%$ & $\mathbf{N}$ & $\%$ \\
\hline & 242 & 100,0 & 242 & 100,0 & 242 & 100,0 \\
\hline Térmico & 16 & 94,1 & 204 & 98,1 & 13 & 76,5 \\
\hline Químico & 1 & 5,9 & 1 & 0,5 & 2 & 11,8 \\
\hline Elétrico & - & - & 3 & 1,4 & 2 & 11,8 \\
\hline TOTAL & 17 & 7,0 & 208 & 85,9 & 17 & 7,0 \\
\hline
\end{tabular}

* Excluídos registros com informações ignoradas.

Tabela 5: Região corpórea atingida pela queimadura, Londrina, PR, Brasil, 2007 a 2011.

\begin{tabular}{|c|c|c|}
\hline \multirow{2}{*}{ Região Corpórea } & n* & $\%$ \\
\hline & 616 & 248,3 \\
\hline Membros superiores & 162 & 65,3 \\
\hline Tronco & 146 & 58,9 \\
\hline Cabeça & 116 & 46,8 \\
\hline Membros inferiores & 99 & 39,9 \\
\hline Pescoço & 56 & 22,6 \\
\hline Genital & 15 & 6,0 \\
\hline Nádega & 14 & 5,6 \\
\hline Vias Aéreas & 8 & 3,2 \\
\hline
\end{tabular}

* Múltiplas regiões atingidas pela queimadura.

Em relação ao desfecho das internações, 58,0\% receberam alta melhorada, 38,0\% alta para reabilitação e 4,0\% faleceram. O tempo médio de internação hospitalar foi de 14,8 dias.

Dos quatro anos e meio analisados, o ano de 2011 foi o que mais apresentou internações com 65 casos $(26,2 \%)$, seguido do ano de 2008 com 60 casos (24,2\%). Em 2007, constataram-se apenas 20 casos, entretanto deve-se considerar que estes dados foram obtidos a partir do mês de agosto, data da inauguração da unidade.

\section{DISCUSSÃo}

A predominância do sexo masculino também foi observada em outras pesquisas nacionais e internacionais $^{(1,4-6,10-13)}$. Normalmente há maior liberdade cultural aos meninos, além de serem menos cautelosos que o sexo oposto, colocando-se em situações de risco com mais frequência ${ }^{(3,10)}$.

Nesta pesquisa a faixa etária de um a três anos se sobressaiu comparado às demais. Estudo que analisou a internação de menores de 13 anos em Unidade de Tratamento de Queimados (UTQ) em São Paulo, entre 2009 e 2010, identificou que a média de idade das crianças atendidas foi de 5,2 anos ${ }^{(14)}$. Na UTQ de
Sergipe, das 526 crianças internadas no período de 2009 a 2010,45,6\% tinham idade inferior a seis anos ${ }^{(15)}$. No Hospital Geral do Andaraí, Rio de Janeiro, de 1997 a 2007, 53,8\% das crianças internadas por queimadura pertenciam à faixa etária de zero a quatro anos ${ }^{(8)}$. Resultados semelhantes também foram encontrado em Lisboa (Portugal) ${ }^{(16)}$ e em Victoria (Austrália) ${ }^{(17)}$. Já, em hospital de referência de João Pessoa, Paraíba, 37\% das vítimas admitidas entre 2007 e 2009 tinham menos de dois anos de idade ${ }^{(18)}$.

A maior incidência dos acidentes na infância é inerente à fase do desenvolvimento neuropsicomotor da criança ainda imaturo, com menor coordenação motora e capacidade cognitiva. Há exigência de maior atenção dos cuidadores adultos quando elas começam a andar, correr, alcançar objetos e brincar em locais impróprios como a cozinha, pois colocam-se constantemente em risco de injúrias ${ }^{(1,3,19)}$.

A maioria dos pacientes deste estudo era procedente de outras regionais de saúde do município de Londrina. Resultados semelhantes foram encontrados em outros hospitais de referência para tratamento de queimados ${ }^{(1-}$ $2,10,12,15,18,20)$. Embora nos últimos anos, no Brasil, tenha aumentado o número de leitos hospitalares especializados nesse agravo em diferentes regiões, ainda 
é insuficiente, especialmente em áreas geográficas extensas como o Norte do país, dificultando o acesso das vítimas aos grandes centros assistenciais ${ }^{(3,10)}$. O custo do tratamento e a manutenção de centros especializados são onerosos para o sistema de saúde comparado à efetiva política pública para a prevenção das queimaduras, que devem ser mais incentivadas e divulgadas no país ${ }^{(21-22)}$.

Nesta pesquisa, a maioria dos acidentes foi registrada nos períodos vespertino e noturno, e nas estações outono/inverno. Estudo nacional recente também identificou esses períodos como os mais frequentes, $36,4 \%$ e $30,8 \%$ respectivamente ${ }^{(23)}$. Já nessas estações do ano acontecem as festas juninas e julinas, meses comemorativos no país, que comumente tem como adereços balões e fogueiras que predispõem aos acidentes com chama direta ${ }^{(15,18)}$. Também há maior frequência de preparo de alimentos quentes com maior risco de escaldaduras, primeira causa de internação hospitalar de crianças em âmbito nacional ${ }^{(1,5,8,11-15,18,24)}$ e internacional $^{(4,16-17)}$.

A escaldadura por líquido superaquecido foi responsável por mais da metade dos acidentes na faixa etária de um a três anos no presente estudo. Entre os maiores de quatro anos predominou chama direta. A chama direta como líquido inflamável, fogo, eletricidade, cinza e incêndio foi a causa mais frequente de internação entre os maiores de cinco anos em hospitais da região metropolitana de Belém (Pará) ${ }^{(13)}$ e de São Paulo(14). Em Lisboa, dos 137 internamentos por queimadura em unidade de cuidados intensivos pediátricos, $38,1 \%$ foi por líquido fervente, $38,1 \%$ por fogo e $23,9 \%$ por eletricidade $^{(16)}$.

A maioria das crianças apresentou queimadura de $2^{\circ}$ grau ou de $20 / 3^{\circ}$ graus nesta pesquisa. A profundidade da lesão depende da temperatura e duração do contato da fonte térmica com a pele ${ }^{(3)}$. Crianças apresentam pele mais sensível que a de um adulto, portanto queimaduras superficiais nestes pacientes podem rapidamente transformar-se em profundas ${ }^{(24)}$. Outros trabalhos identificaram resultados semelhantes, especialmente entre os menores de dois anos de idade pela fragilidade epidérmica, favorecendo maior extensão e profundidade da lesão, com consequente sequelas física e motora ${ }^{(1,3-4,8,10-11,14,16,24)}$.

Em relação à região corpórea atingida pela queimadura nas crianças, observou-se maior prevalência nos membros superiores, seguida pelo tronco. Outros estudos, em Minas Gerais e São Paulo, convergem com esses achados ${ }^{(2,14)}$. Essas regiões são mais acometidas dadas as circunstâncias em que a maioria dos acidentes ocorrem, ou seja, alcançando líquidos quentes no fogão, por exemplo, ou manipulando materiais inflamáveis que representam risco de queimaduras por chama direta.

O tempo médio de internação hospitalar no presente estudo foi de 14,8 dias. Em hospital de Sergipe observou-se resultado semelhante ${ }^{(15)}$. Já em João Pessoa (Paraíba) foi de 5,87 dias $^{(18)}$. O período de hospitalização pode variar de dias a meses, dependendo da extensão e profundidade da lesão, e presença de complicações como infecção e gravidade do caso, favorecendo maior tempo em unidades de terapia intensiva ${ }^{(4,7,13,16,24)}$.

Número importante de crianças recebeu alta com necessidade de reabilitação com fisioterapeutas, psicólogos e/ou assistentes sociais, além de retornos às consultas médicas ambulatoriais. Também se observou mortes por complicações da queimadura em ambiente hospitalar. Percentual semelhante de óbito foi encontrado em Sergipe $(3,1 \%)^{(16)}$, na capital paulista $(5,9 \%)^{(25)}$ e em Lisboa $(8,1 \%)^{(16)}$.

Em virtude da gravidade e complexa recuperação da criança vítima de queimadura, tanto em países desenvolvidos como em desenvolvimento, ressalta-se a importância de pesquisas epidemiológicas na temática por poderem respaldar estratégias preventivas, em especial no período da infância. Esse grupo etário está exposto às maiores complicações com consequente elevação nas taxas de morbidade e mortalidade por causas evitáveis ${ }^{(3-4,7,10,13,21)}$. Por outro lado, há necessidade de mais investimentos na qualificação de recursos humanos e implantação de serviços especializados para efetiva implementação de ações de prevenção, promoção e reabilitação.

\section{CONCLUSÃO}

Os resultados desta pesquisa mostraram que houve maior frequência de queimaduras entre os meninos, no período vespertino e nas estações outono/inverno. A escaldadura foi o fortuito mais ocorrido entre as crianças pequenas, e a chama direta foi mais frequente entre as mais velhas. Os locais mais acometidos foram membros superiores, tronco e cabeça, o tempo médio de internação foi de aproximadamente duas semanas, e importante percentual das crianças necessitou de reabilitação após a alta hospitalar.

Este estudo expôs o grande número de infantes envolvidos em acidentes por queimaduras e a necessidade de conscientização sobre o tema por meio 
de programas educativos em escolas, creches e unidades de saúde. Os responsáveis pelas crianças devem estar cientes sobre a vulnerabilidade infantil e os contextos mais frequentes de ocorrência de queimadura. Além

\section{REFERÊNCIAS}

1. Dassie LTD, Alves EONM. Centro de tratamento de queimados: perfil epidemiológico de crianças internadas em um hospital escola. Rev Bras Queimaduras. 2011;10(1):10-4.

2. Montes SF, Barbosa MH, Sousa Neto AL. Aspectos clínicos e epidemiológicos de pacientes queimados internados em um Hospital de Ensino. Rev Esc Enferm USP. 2011; 45(2):369-73

3. Pereima M. Particularidades de Queimaduras em Crianças. In: Lima Junior EML, Novaes FN, Piccolo N, Serra MCVF. Tratado de Queimaduras no Paciente Agudo. 2a Ed. São Paulo: Atheneu; 2009. p.509-19.

4. D'Souza AL, Nelson NG, McKenzie LB. Pediatric burn injuries treated in US emergency departments between 1990 and 2006. Pediatrics. 2009; 124:1424-30.

5. DATASUS [internet]. Brasília: Ministério da Saúde (BR) [ acesso em 07 maio 2014]. Internações por faixa etária segundo causas externas relacionadas a queimadura. Morbidade hospitalar do Sistema Único de Saúde por causas externas, 1997 a 2007. Disponível em:

http://tabnet.datasus.gov.br/cgi/deftohtm.exe?sih/cnv/eruf.def. 6. DATASUS [internet]. Brasília: Ministério da Saúde (BR) [acesso em 07 maio 2014]. Óbitos p/ Residência por Faixa Etária segundo Região

Período: 1996-2011.Disponível

em: http://tabnet.datasus.gov.br/cgi/tabcgi.exe?sim/cnv/ext10uf. def 1996 a 2011

7. Viana FP, Resende SM, Tolêdo MC, Silva RC. Aspectos epidemiológicos das crianças com queimaduras internadas no Pronto Socorro para Queimaduras de Goiânia - Goiás. Rev. Eletr. Enf. [Internet]. 2009; 11(4):779-84. Available from:

http://www.fen.ufg.br/revista/v11/n4/v11n4a02.htm. 8. Machado THS et al. Estudo epidemiológico das crianças queimadas de 0-15 anos atendidas no Hospital Geral do Andaraí, durante o período de 1997 a 2007. Rev Bras Queimaduras. 2009; 8(1):3-8.

9. Silva GPF, Olegario NBC, Pinheiro AMRS, Bastos VPD. Estudo epidemiológico dos pacientes idosos queimados no Centro de Tratamento de Queimados do Hospital Instituto Doutor José Frota do município de Fortaleza-CE, no período de 2004 a 2008. Rev Bras Queimaduras. 2010; 9(1):7-10.

10. Herson MR, Teixeira Neto N, Paggiaro AO, Carvalho VF, Machado LCC, Ueda $T$, et al. Estudo epidemiológico das sequelas de queimaduras: 12 anos de experiência da Unidade de Queimaduras da Divisão de Cirurgia Plástica do Hospital das Clínicas da Faculdade de Medicina da USP. Rev Bras Queimaduras. 2009; 8(3):82-6.

11. Yoda CN, Leonardi DF, Feijó R. Queimadura pediátrica: fatores associados a sequelas físicas em crianças queimadas atendidas no Hospital Infantil Joana de Gusmão. Rev Bras Queimaduras. 2013; 12(2):112-7.

12. Mendes CA, Sá DM, Padovese SM, Cruvinel SS. Estudo epidemiológico de queimaduras atendidas nas Unidades de Atendimento Integrado de Uberlândia-MG entre 2000 a 2005. Rev Bras Queimaduras. 2009; 8(1):18-22.

13. Oliveira FPS, Ferreira EAP, Carmona SS. Crianças e adolescentes vítimas de queimaduras: caracterização de situações de risco ao desenvolvimento. Rev Bras Crescimento Desenvolvimento Hum. 2009; 19(1):19-34.

14. Millan LS, Gemperli R, Tovo FM, Mendaçolli TJ, Gomez DS, Ferreira MC. Estudo epidemiológico de queimaduras em crianças atendidas em hospital terciário na cidade de São Paulo. Rev Bras Cir Plást. 2012; 27(4):611-5

15. Moreira CA, Reis IF, Melo-Costa ACS. Perfil epidemiológico de pacientes atendidos na unidade de tratamento de queimados no município de Aracaju (SE). Cad. Graduação - ciênc. biol. e da disso, há necessidade de investimentos em centros de tratamento especializados com recursos humanos qualificados para melhor acompanhamento e prognóstico dos pacientes.

saúde. $2011 ; 13(14): 111-27$.

16. Francisco $T$, Nóbrega $S$, Valente $R$, Santos M, Pereira G, Estrada J et al. Grande queimado numa Unidade de Cuidados Intensivos Pediátricos: experiência de 20 anos. Nascer e Crescer. 2013; 22(3):151-57.

17. Wasiak J, Spinks A, Ashby K, Clapperton A, Cleland H, Gabbe $B$. The epidemiology of burn injuries in an Australian setting, 2000-2006. Burns. 2009; 35(8):1124-32.

18. Fernandes FMFA, Torquato IMB, Dantas MAS, Pontes Júnior FAC, Ferreira JA, Collet N. Queimaduras em crianças e adolescentes: caracterização clínica e epidemiológica. Rev Gaúcha Enferm. 2012;33(4):133-41.

19. Coutinho BBA, Balbuena MB, Anbar RA, Anbar RA, Almeida KG, Almeida PYNG. Perfil epidemiológico de pacientes internados na enfermaria de queimados da Associação Beneficente de Campo Grande Santa Casa/MS. Rev. Bras. Cir. Plást. (Impr.) 2010;25(4):600-603.

20. Gimenes GA, Alferes FCBA, Dorsa PP, Barros ACP, Gonella HA. Estudo epidemiológico de pacientes internados no Centro de Tratamento de Queimados do Conjunto Hospitalar de Sorocaba Rev Bras Queimaduras. 2009;8(1):14-7.

21. Takejima ML, Netto RFB, Toebe BL, Andretta MA, Prestes MA, Takaki JL et al. Prevenção de queimaduras: avaliação de conhecimento sobre prevenção de queimaduras em usuários das unidades de saúde de Curitiba. Rev Bras Queimaduras. 2011; $10(3): 85-8$.

22. Soares NTI, Tacla MTGM. Experience of nursing staff facing the hospitalization of burned children. Invest Educ Enferm. 2014; 32(1)49-59.

23. Gawryszewski VP et AL. Atendimentos decorrentes de queimaduras em serviços públicos de emergência no Brasil, 2009. Cad. Saúde Pública. 2012; 28(4):629-40.

24. Santana VBRL. Perfil epidemiológico de crianças vítimas de queimaduras no Município de Niterói-RJ. Rev Bras Queimaduras. 2010; 9(4):136-9.

25. Lacerda LA, Carneiro AC, Oliveira AF, Ferreira AGLM. Estudo epidemiológico da Unidade de Tratamento de Queimaduras da Universidade Federal de São Paulo. Rev Bras Queimaduras. 2010; 9(3):82-8.

Artigo recebido em 04/04/2013. Aprovado para publicação em 23/04/2014. Artigo publicado em 30/09/2014. 